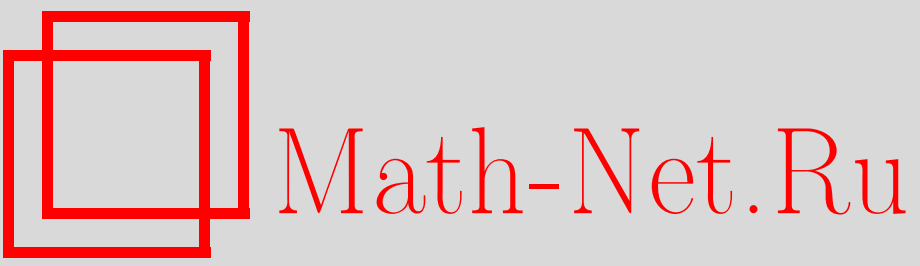

S. Vakeroudis, On hitting times of the winding processes of planar Brownian motion and of Ornstein-Uhlenbeck processes, via Bougerol's identity, Теория вероятн. и ее примен., 2011, том 56, выпуск 3, 566-591

DOI: https://doi.org/10.4213/tvp4407

Использование Общероссийского математического портала Math-Net.Ru подразумевает, что вы прочитали и согласны с пользовательским соглашением

http://www. mathnet.ru/rus/agreement

Параметры загрузки:

IP: 54.196 .121 .252

26 апреля 2023 г., 03:10:14 
2011

(c) 2011 г.

VAKEROUDIS S.*

\title{
ON HITTING TIMES OF THE WINDING PROCESSES OF PLANAR BROWNIAN MOTION AND OF ORNSTEIN-UHLENBECK PROCESSES, VIA BOUGEROL'S IDENTITY
}

\begin{abstract}
Устанавливаются некоторые тождества по распределению для плоских комплекснозначных процессов Орнштейна-Уленбека $\left(Z_{t}=\right.$ $\left.X_{t}+i Y_{t}, t \geqslant 0\right)$, включая плоское броуновское движение, эквивалентные хорошо известному тождеству Бужероля для линейного броуновского движения $\left(\beta_{t}, t \geqslant 0\right)$ : для любого фиксированного $u>0$

$$
\operatorname{sh} \beta_{u} \stackrel{\text { law }}{=} \hat{\beta}_{\int_{0}^{u}}^{u} d s \exp \left(2 \beta_{s}\right)
$$

где $\left(\hat{\beta}_{t}, t \geqslant 0\right)$ - броуновское движение, независимое от $\beta$.

Эти тождества по распределению для двумерных процессов позволяют изучать распределение моментов достижения $T_{c}^{\theta} \equiv$ $\inf \left\{t: \theta_{t}=c\right\}(c>0), T_{-d, c}^{\theta} \equiv \inf \left\{t: \theta_{t} \notin(-d, c)\right\}(c, d>0)$ и, в частности, $T_{-c, c}^{\theta} \equiv \inf \left\{t: \theta_{t} \notin(-c, c)\right\} \quad(c>0)$ для непрерывного процесса $\theta_{t}=\operatorname{Im}\left(\int_{0}^{t} Z_{s}^{-1} d Z_{s}\right), t \geqslant 0,-$ угла, заметаемого комплексным процессом Орнштейна-Уленбека.

Ключевые слова и фразы: плоское броуновское движение; процесс Орнштейна-Уленбека; угол, заметаемый радиус-вектором комплекснозначного процесса; тождество Бужероля; момент выхода из конуса.
\end{abstract}

1. Introduction. The conformal invariance of planar Brownian motion has deep consequences as to the structure of its trajectories (see, e.g., [20]). In particular, a number of articles have been devoted to the study of its continuous winding process $\left(\theta_{t}, t \geqslant 0\right)$ : [32], [34], [15], [27], [29], [21], [5], [37], [28], [4]. In this paper, we take up again the study of the first hitting times:

$$
T_{-d, c}^{\theta} \equiv \inf \left\{t: \theta_{t} \notin(-d, c)\right\} \quad(c, d>0),
$$

*Laboratoire de Probabilités et Modèles Aléatoires (LPMA) CNRS: UMR7599, Université Pierre et Marie Curie-Paris VI, 4 Place Jussieu, 75252 Paris Cedex 05, France; Département de Mathématiques et de Biologie CNRS: UMR8544, Ecole Normale Supérieure, 46 rue d'Ulm, 75005 Paris, France; e-mail: stavros.vakeroudis@etu.upmc.fr 
this time in relation with Bougerol's well-known identity (see [8], [2], and [38]): for fixed $u>0$,

$$
\operatorname{sh} \beta_{u} \stackrel{\text { law }}{=} \hat{\beta} \int_{0}^{u} d s \exp \left(2 \beta_{s}\right),
$$

where $\left(\hat{\beta}_{t}, t \geqslant 0\right)$ is a Brownian motion ${ }^{1)}$ independent of $\beta$. In particular, it turns out that, for fixed $c>0$,

$$
\theta_{T_{c}^{\hat{\beta}}} \stackrel{\text { law }}{=} C_{a(c)},
$$

where $\hat{\beta}$ is a Brownian motion independent of $\left(\theta_{u}, u \geqslant 0\right), T_{c}^{\hat{\beta}}=\inf \left\{t: \hat{\beta}_{t}=\right.$ $c\},\left(C_{t}, t \geqslant 0\right)$ is a standard Cauchy process and $a(c)=\arg \operatorname{sh} c \equiv \ln (c+$ $\left.\sqrt{1+c^{2}}\right), c \in \mathbf{R}$.

The identity $(\star)$ yields yet another proof of the celebrated Spitzer theorem:

$$
\frac{2}{\ln t} \theta_{t} \underset{t \rightarrow \infty}{\stackrel{\text { law }}{\longrightarrow}} C_{1}
$$

with the help of Williams' «pinching method» (see [34] and [27]).

Moreover, we study the distributions of $T_{-\infty, c}^{\theta}$ and $T_{-c, c}^{\theta}$. In particular, we give explicit formulae for the density function of $T_{-c, c}^{\theta}$ and for the first moment of $\ln T_{-c, c}^{\theta}$.

The last section of the paper is devoted to developing similar results when planar Brownian motion is replaced by a complex valued OrnsteinUhlenbeck process. We note that Bertoin and Werner [5] already made discussions of windings for planar Brownian motion using arguments related to Ornstein-Uhlenbeck processes.

Firstly, we obtain some analogue of $(\star)$ when $T_{c}^{\hat{\beta}}$ is replaced by $T_{c}^{(\lambda)}=$ $T_{-c, c}^{\theta^{Z}}=\inf \left\{t:\left|\theta_{t}^{Z}\right|=c\right\}$, the corresponding time for an Ornstein-Uhlenbeck process with parameter $\lambda$. Secondly, we identify the distribution of $T_{c}^{(\lambda)}$. More specifically, we derive the asymptotics of $\mathbf{E}\left[T_{c}^{(\lambda)}\right]$ for $\lambda$ large and for $\lambda$ small.

\section{The Brownian motion case.}

2.1. A reminder on planar Brownian motion. Let $\left(Z_{t}=X_{t}+\right.$ $\left.i Y_{t}, t \geqslant 0\right)$ denote a standard planar Brownian motion, starting from $x_{0}+i 0$, $x_{0}>0$, where $\left(X_{t}, t \geqslant 0\right)$ and $\left(Y_{t}, t \geqslant 0\right)$ are two independent linear Brownian motions, starting, respectively, from $x_{0}$ and 0 .

It is well known (see, e.g., [19]) that, since $x_{0} \neq 0$, the process $\left(Z_{t}, t \geqslant 0\right)$ does not visit a.s. the point 0 but keeps winding around 0 infinitely often.

1) When we simply write: Brownian motion, we always mean real-valued Brownian motion starting from 0. For 2-dimensional Brownian motion, we add qualifier planar or complex. 
In particular, the continuous winding process $\theta_{t}=\operatorname{Im}\left(\int_{0}^{t} Z_{s}^{-1} d Z_{s}\right), t \geqslant 0$, is well defined.

Furthermore, there is the skew-product representation:

$$
\ln \left|Z_{t}\right|+i \theta_{t} \equiv \int_{0}^{t} \frac{d Z_{s}}{Z_{s}}=\left.\left(\beta_{u}+i \gamma_{u}\right)\right|_{u=H_{t}=\int_{0}^{t}\left|Z_{s}\right|^{-2} d s}
$$

where $\left(\beta_{u}+i \gamma_{u}, u \geqslant 0\right)$ is another planar Brownian motion starting from $\ln x_{0}+i 0$. For a study of the Bessel clock $H$, see [35].

Rewriting (1) as

$$
\ln \left|Z_{t}\right|=\beta_{H_{t}}, \theta_{t}=\gamma_{H_{t}},
$$

we easily obtain that the total $\sigma$-fields $\sigma\left\{\left|Z_{t}\right|, t \geqslant 0\right\}$ and $\sigma\left\{\beta_{u}, u \geqslant 0\right\}$ are identical, whereas $\left(\gamma_{u}, u \geqslant 0\right)$ is independent from $\left(\left|Z_{t}\right|, t \geqslant 0\right)$.

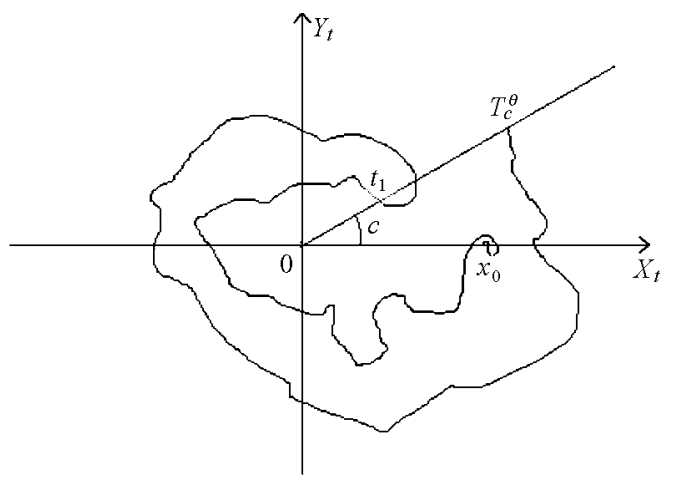

Fig. 1. Exit time from a single border cone for a planar BM. This figure presents the exit times $T_{c}^{\theta}\left(t_{1}\right.$ does not matter because the angle is negative).

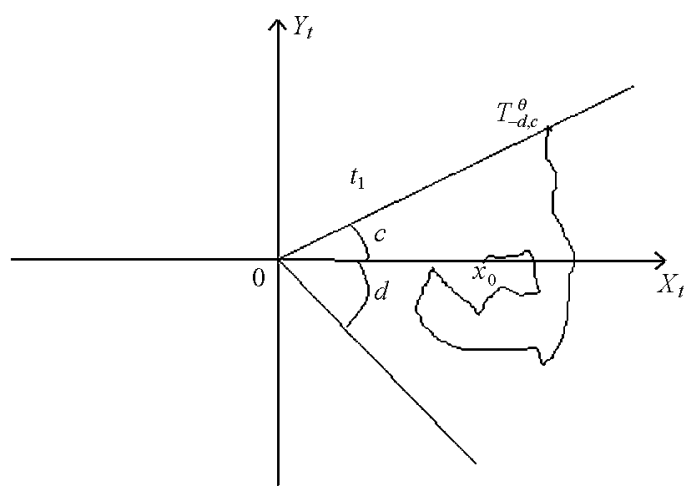

Fig. 2. Exit time from a double border cone for a planar BM. This figure presents the exit times $T_{-d, c}^{\theta}$ for a planar BM starting from $x_{0}+i 0$. 
A number of studies of the properties of the first hitting time (see Figure 2)

$$
T_{-d, c}^{\theta} \equiv \inf \left\{t: \theta_{t} \notin(-d, c)\right\} \quad(c, d>0),
$$

have been developed, going back to [32].

In particular, it is well known ([32], [9], [31, Exercise 2.21, p. 196]) that

$$
\mathbf{E}\left[\left(T_{-d, c}^{\theta}\right)^{p}\right]<\infty \quad \text { if and only if } \quad p<\frac{\pi}{2(c+d)} .
$$

Moreover, Spitzer's asymptotic theorem (see, e.g., [32]) states that

$$
\frac{2 \theta_{t}}{\ln t} \underset{t \rightarrow \infty}{\stackrel{\text { law }}{\longrightarrow}} C_{1} \stackrel{\text { law }}{=} \gamma_{T_{1}^{\beta}}
$$

where $C_{1}$ is a standard Cauchy variable.

2.2. On the Laplace transform of the distribution of the hitting time $T_{c}^{\theta} \equiv T_{-\infty, c}^{\theta}$. Now, we use the representation (2) to access the distribution of $T_{c}^{\theta}$ (see Figure 1). We define $T_{c}^{\gamma} \equiv \inf \left\{t: \gamma_{t} \notin(-\infty, c)\right\}$ the hitting time associated to the Brownian motion $\left(\gamma_{t}, t \geqslant 0\right)$. Note that from (2) it follows that $H_{T_{c}^{\theta}}=T_{c}^{\gamma}$, hence $T_{c}^{\theta}=\left.H_{u}^{-1}\right|_{u=T_{c}^{\gamma}}$, where

$$
H_{u}^{-1} \equiv \inf \left\{t: H_{t}>u\right\}=\int_{0}^{u} d s \exp \left(2 \beta_{s}\right) \equiv A_{u} .
$$

Thus, we have obtained

$$
T_{c}^{\theta}=A_{T_{c}^{\gamma}},
$$

where $\left(A_{u}, u \geqslant 0\right)$ and $T_{c}^{\gamma}$ are independent, since $\beta$ and $\gamma$ are independent. We can write $\beta_{s}=\ln x_{0}+\beta_{s}^{(0)}$, with $\left(\beta_{s}^{(0)}, s \geqslant 0\right)$ a standard one-dimensional Brownian motion starting from 0 . Then, we deduce from (6) that

$$
T_{c}^{\theta}=x_{0}^{2}\left(\int_{0}^{T_{c}^{\gamma}} d s \exp \left(2 \beta_{s}^{(0)}\right)\right) .
$$

From now on, for simplicity, we shall take $x_{0}=1$, but this is really no restriction, as the dependency in $x_{0}$, which is exhibited in (7), is very simple. We shall also make use of Bougerol's identity ([8], [2], and [38, p. 200]) which is very useful to study the distribution of $A_{u}$ (see, e.g., [25], [26]). For any fixed $u>0$

$$
\operatorname{sh} \beta_{u} \stackrel{\text { law }}{=} \hat{\beta}_{A_{u}}=\hat{\beta}_{\int_{0}^{u} d s \exp \left(2 \beta_{s}\right)},
$$

where on the right-hand side, $\left(\hat{\beta}_{t}, t \geqslant 0\right)$ is a Brownian motion independent of $A_{u} \equiv \int_{0}^{u} d s \exp \left(2 \beta_{s}\right)$. 
Thus, from (8) and (6), and as is well known (see, e.g., [31]), the law of $\beta_{T_{c}^{\gamma}}$ is the Cauchy law with parameter $c$, i.e., with density

$$
h_{c}(y)=\frac{c}{\pi\left(c^{2}+y^{2}\right)},
$$

we deduce the following proposition.

Proposition 2.1. For fixed $c>0$, there is the following identity in law:

$$
\operatorname{sh} C_{c} \stackrel{\text { law }}{=} \hat{\beta}_{T_{c}^{\theta}},
$$

where, on the left hand side, $\left(C_{c}, c \geqslant 0\right)$ denotes a standard Cauchy process and on the right-hand side, $\left(\hat{\beta}_{u}, u \geqslant 0\right)$ is a one-dimensional Brownian motion, independent from $T_{c}^{\theta}$.

We may now identify the densities of the variables found on both sides of (9), i.e.,

on the left-hand side: $\frac{1}{\sqrt{1+x^{2}}} h_{c}(\arg \operatorname{sh} x)=\frac{1}{\sqrt{1+x^{2}}} h_{c}(a(x))$,

on the right-hand side: $\mathbf{E}\left[\frac{1}{\sqrt{2 \pi T_{c}^{\theta}}} \exp \left(-\frac{x^{2}}{2 T_{c}^{\theta}}\right)\right]$,

where $a(x)=\arg \operatorname{sh} x$.

Thus, we have obtained the following proposition.

Proposition 2.2. The distribution of $T_{c}^{\theta}$ may be characterized by

$$
\mathbf{E}\left[\frac{1}{\sqrt{2 \pi T_{c}^{\theta}}} \exp \left(-\frac{x}{2 T_{c}^{\theta}}\right)\right]=\frac{1}{\sqrt{1+x}} \frac{c}{\pi\left(c^{2}+\ln ^{2}(\sqrt{x}+\sqrt{1+x})\right)},
$$

for $x \geqslant 0$.

The proof of Proposition 2.2 follows from the identity $a(y)=\arg \operatorname{sh} y \equiv$ $\ln \left(y+\sqrt{1+y^{2}}\right)$ and by making the change of variable $y^{2}=x$. Let us now define the probability

$$
\mathbf{Q}_{c}=\sqrt{\frac{\pi c^{2}}{2 T_{c}^{\theta}}} \cdot \mathbf{P} .
$$

The fact that $\mathbf{Q}_{c}$ is a probability follows from (10) by taking $x=0$. Thus we obtain that $c \mathbf{E}\left[\sqrt{\pi /\left(2 T_{c}^{\theta}\right)}\right]=1$, and we can write

$$
\mathbf{E}_{\mathbf{Q}_{c}}\left[\exp \left(-\frac{x}{2 T_{c}^{\theta}}\right)\right]=\frac{1}{\sqrt{1+x}} \frac{1}{1+c^{-2} \ln ^{2}(\sqrt{x}+\sqrt{1+x})},
$$

for $\forall x \geqslant 0$, which yields the Laplace transform of $1 / T_{c}^{\theta}$ under $\mathbf{Q}_{c}$.

Let us now take a look at what happens if we make $c$ tend to $\infty$. Denote by $T_{1}^{\beta} \equiv \inf \left\{t: \beta_{t}=1\right\}$ the first hitting time of level 1 for a standard 
Brownian motion $\beta$ and by $N$ a standard Gaussian variable $\mathscr{N}(0,1)$. Then, from equation (11), we obtain

$$
\lim _{c \rightarrow \infty} \mathbf{E}_{\mathbf{Q}_{c}}\left[e^{-x /\left(2 T_{c}^{\theta}\right)}\right]=\mathbf{E}\left[e^{-x N^{2} / 2}\right]=\mathbf{E}\left[e^{-x /\left(2 T_{1}^{\beta}\right)}\right],
$$

which means that $T_{c}^{\theta} \stackrel{\text { law }}{\longrightarrow} T_{1}^{\beta}$ as $c \rightarrow \infty$. (At this point, one may wonder whether there is some kind of convergence in law involving $\left(\theta_{u}, u \geqslant 0\right)$, under $\mathbf{Q}_{c}$, as $c \rightarrow \infty$, but we shall not touch this point.)

Proposition 2.2 has the following consequence.

Corollary 2.1. Let $\varphi(x)$ denote the Laplace transform (11), that is the Laplace transform of $1 /\left(2 T_{c}^{\theta}\right)$ under $\mathbf{Q}_{c}$. Then, the Laplace transform of $1 /\left(2 T_{c}^{\theta}\right)$ under $\mathbf{P}$ is

$$
\mathbf{E}\left[\exp \left(-\frac{x}{2 T_{c}^{\theta}}\right)\right]=\int_{x}^{\infty} \frac{d w}{\sqrt{w-x}} \varphi(w) .
$$

P r o o f. By Fubini's theorem, we deduce from (11) that

$$
\begin{aligned}
\mathbf{E}\left[\exp \left(-\frac{x}{2 T_{c}^{\theta}}\right)\right] & =\int_{0}^{\infty} \frac{d y}{\sqrt{y}} \mathbf{E}\left[\frac{1}{\sqrt{2 \pi T_{c}^{\theta}}} \exp \left(-\frac{x+y}{2 T_{c}^{\theta}}\right)\right] \\
& =\int_{0}^{\infty} \frac{d y}{\sqrt{y}} \varphi(x+y) \stackrel{y=x t}{=} \sqrt{x} \int_{0}^{\infty} \frac{d t}{\sqrt{t}} \varphi(x(1+t)) \\
& \stackrel{v=1+t}{=} \sqrt{x} \int_{1}^{\infty} \frac{d v}{\sqrt{v-1}} \varphi(x v) \stackrel{w=x v}{=} \int_{x}^{\infty} \frac{d w}{\sqrt{w-x}} \varphi(w),
\end{aligned}
$$

which is formula (13).

2.3. Some related identities in law. This subsection is strongly related to [14]. A slightly different look at the combination of Bougerol's identity (8) and the skew-product representation (1) leads to the following striking identities in law.

Proposition 2.3. Let $\left(\delta_{u}, u \geqslant 0\right)$ be a one-dimensional Brownian motion independent of the planar Brownian motion $\left(Z_{u}, u \geqslant 0\right)$ starting from $1+i 0$. Then, for any $b \geqslant 0$, the following identities in law hold:
(i) $H_{T_{b}^{\delta}} \stackrel{\text { law }}{=} T_{a(b)}^{\beta}$,
(ii) $\theta_{T_{b}^{\delta}} \stackrel{\text { law }}{=} C_{a(b)}$,
(iii) $\bar{\theta}_{T_{b}^{\delta}} \stackrel{\text { law }}{=}\left|C_{a(b)}\right|$

where $C_{A}$ is a Cauchy variable with parameter $A$ and $\bar{\theta}_{u}=\sup _{s \leqslant u} \theta_{s}$.

P r o of. By the symmetry principle (see [3] for the original note and [16] for a detailed discussion), Bougerol's identity can be stated equivalently as

$$
\operatorname{sh} \bar{\beta}_{u} \stackrel{\text { law }}{=} \bar{\delta}_{A_{u}(\beta)} .
$$


Consequently, the laws of the first hitting times of a fixed level $b$ by the processes on each side of (14) are identical, that is,

$$
T_{a(b)}^{\beta} \stackrel{\text { law }}{=} H_{T_{b}^{\delta}},
$$

which is (i).

(ii) follows from (i), since

$$
\theta_{u} \stackrel{\text { law }}{=} \gamma_{H_{u}}
$$

where $\left(\gamma_{s}, s \geqslant 0\right)$ is a Brownian motion independent of $\left(H_{u}, u \geqslant 0\right)$ and $\left(C_{u}, u \geqslant 0\right)$ can be represented as $\left(\gamma_{T_{u}^{\beta}}, u \geqslant 0\right)$.

(iii) follows from (ii), again with the help of the symmetry principle.

$\mathrm{R}$ e $\mathrm{m}$ a $\mathrm{r}$ k. Proposition 2.2 can also be derived from (iii) in Proposition 2.3. Indeed, for $c>0$, starting from the left-hand side of (iii), and letting $N \sim \mathscr{N}(0,1)$ independent from $T_{c}^{\theta}$ :

$$
\begin{aligned}
\mathbf{P}\left(\bar{\theta}_{T_{b}^{\delta}}<c\right) & =\mathbf{P}\left(T_{b}^{\delta}<T_{c}^{\theta}\right)=\mathbf{P}\left(b<\bar{\delta}_{T_{c}^{\theta}}\right) \\
& =\mathbf{P}\left(b<\sqrt{T_{c}^{\theta}}|N|\right)=\mathbf{P}\left(\frac{b}{\sqrt{T_{c}^{\theta}}}<|N|\right) \\
& =\sqrt{\frac{2}{\pi}} \mathbf{E}\left[\int_{b / \sqrt{T_{c}^{\theta}}}^{\infty} d y e^{\left.-y^{2} / 2\right],}\right.
\end{aligned}
$$

while, on the right-hand side of (iii):

$$
\mathbf{P}\left(\left|C_{a(b)}\right|<c\right)=2 \int_{0}^{c} \frac{a(b) d y}{\pi\left(a^{2}(b)+y^{2}\right)} \stackrel{y=a(b) h}{=} \frac{2}{\pi} \int_{0}^{c / a(b)} \frac{d h}{1+h^{2}} .
$$

Taking derivatives in (15) and (16) with respect to $b$ and changing the variables $b=\sqrt{x}$, we obtain Proposition 2.2.

2.4. Recovering Spitzer's theorem. The identity (ii) in Proposition 2.3 is reminiscent of Williams' remark (see [34], [27]), that:

$$
H_{T_{r}^{R}} \stackrel{\text { law }}{=} T_{\ln r}^{\delta},
$$

where $R$ starts from 1 and $\delta$ starts from 0 (in fact, this is a consequence of (2)). For a number of variants of (17), see [36], [24]. This was D. Williams' starting point for a noncomputational proof of Spitzer's result (4). We note that in (ii), $T_{b}^{\delta}$ is independent of the process $\left(\theta_{u}, u \geqslant 0\right)$ while in (17) $T_{r}^{R}$ depends on $\left(\theta_{u}, u \geqslant 0\right)$. Actually, we can mimic Williams' «pinching method» to derive Spitzer's theorem (4) from (ii) in Proposition 2.3.

Proposition 2.4 (A new proof of the Spitzer theorem). As $t \rightarrow \infty$, $\theta_{T_{\sqrt{t}}^{\delta}}-\theta_{t}$ converges in law, which implies that

$$
\frac{1}{\ln t}\left(\theta_{T_{\sqrt{t}}^{\delta}}-\theta_{t}\right) \underset{t \rightarrow \infty}{\stackrel{\mathbf{P}}{\longrightarrow}} 0
$$


and, in turn, implies Spitzer's theorem (see formula (4)):

$$
\frac{2}{\ln t} \theta_{t} \underset{t \rightarrow \infty}{\stackrel{\text { law }}{\longrightarrow}} C_{1}
$$

P r o o f. From equation (ii) of Proposition 2.3 we note that

$$
\frac{1}{\ln b} \theta_{T_{b}^{\delta}} \stackrel{\text { law }}{=} \frac{C_{a(b)}}{\ln b} \underset{b \rightarrow \infty}{\stackrel{\text { law }}{\longrightarrow}} C_{1} .
$$

So, for $b=\sqrt{t}$ we have

$$
\frac{2}{\ln t} \theta_{T_{\sqrt{t}}^{\delta}} \underset{b \rightarrow \infty}{\stackrel{\text { law }}{\longrightarrow}} C_{1} .
$$

On the other hand, following Williams' «pinching method», we note that

$$
\frac{1}{\ln t}\left(\theta_{T_{\sqrt{t}}^{\delta}}-\theta_{t}\right) \underset{t \rightarrow \infty}{\stackrel{\text { law }}{\longrightarrow}} 0
$$

since $Z_{u}=x_{0}+Z_{u}^{(0)}$ and also, by making the change of variables $u=t v$ and using the scaling property, we obtain

$$
\theta_{T_{\sqrt{t}}^{\delta}}-\theta_{t} \equiv \operatorname{Im}\left(\int_{t}^{T_{\sqrt{t}}^{\delta}} \frac{d Z_{u}}{Z_{u}}\right) \underset{t \rightarrow \infty}{\stackrel{\operatorname{law}}{\longrightarrow}} \operatorname{Im}\left(\int_{1}^{T_{1}^{\delta}} \frac{d Z_{v}^{(0)}}{Z_{v}^{(0)}}\right) .
$$

Here, the limit variable is, in our opinion, of no other interest than its existence which implies (18), hence (4).

2.5. On the distributions of $T_{c}^{\theta} \equiv T_{-\infty, c}^{\theta}$ and $T_{-c, c}^{\theta}$.

Proposition 2.5. The following asymptotic equivalence holds:

$$
(\ln t) \mathbf{P}\left(T_{c}^{\theta}>t\right) \underset{t \rightarrow \infty}{\longrightarrow} \frac{4 c}{\pi} .
$$

As a consequence, for $\eta>0, \mathbf{E}\left[\left(\ln T_{c}^{\theta}\right)_{+}^{\eta}\right]<\infty$ if and only if $\eta<1$ (where $(\cdot)_{+}$denotes the positive part $)$.

P r o of. a) We rely upon the asymptotic distribution of $H_{t} \equiv$ $\int_{0}^{t}\left|Z_{s}\right|^{-2} d s$ which is given by [31]:

$$
\frac{4 H_{t}}{(\ln t)^{2}} \underset{t \rightarrow \infty}{\stackrel{\text { law }}{\longrightarrow}} T_{1}^{\beta} \equiv \inf \left\{t: \beta_{t}=1\right\}
$$

or, equivalently,

$$
\frac{\ln t}{2 \sqrt{H_{t}}} \underset{t \rightarrow \infty}{\stackrel{\text { law }}{\longrightarrow}}|N|,
$$

where $N$ is a standard Gaussian variable $\mathscr{N}(0,1)$. 
We note that, by the representation (2) of $\theta_{t}$, the result (20) is equivalent to Spitzer's theorem [32]:

$$
\frac{2 \theta_{t}}{\ln t} \underset{t \rightarrow \infty}{\stackrel{\text { law }}{\longrightarrow}} C_{1} \stackrel{\text { law }}{=} \gamma_{T_{1}^{\beta}}
$$

where $C_{1}$ is a standard Cauchy variable.

b) We shall now use this, in order to deduce Proposition 2.5. We denote $S_{t}^{\theta} \equiv \sup _{s \leqslant t} \theta_{s} \equiv S_{H_{t}}^{\gamma}$ and we note that (from scaling)

$$
\mathbf{P}\left(T_{c}^{\theta} \geqslant t\right)=\mathbf{P}\left(S_{H_{t}}^{\gamma} \leqslant c\right)=\mathbf{P}\left(\sqrt{H_{t}} S_{1}^{\gamma} \leqslant c\right),
$$

since $\gamma$ and $H$ are independent. Thus, we have (since $S_{1}^{\gamma} \stackrel{\text { law }}{=}|N|$ and by making the change of variable $\left.x=c y / \sqrt{H_{t}}\right)$ :

$$
\begin{aligned}
\mathbf{P}\left(T_{c}^{\theta} \geqslant t\right) & =\sqrt{\frac{2}{\pi}} \mathbf{E}\left[\int_{0}^{c / \sqrt{H_{t}}} d x e^{-x^{2} / 2}\right] \\
& =\sqrt{\frac{2}{\pi}} c \mathbf{E}\left[\int_{0}^{1} \frac{d y}{\sqrt{H_{t}}} \exp \left(-\frac{c^{2} y^{2}}{2 H_{t}}\right)\right] .
\end{aligned}
$$

We now deduce from (21) that

$$
\frac{\ln t}{2} \mathbf{P}\left(T_{c}^{\theta} \geqslant t\right) \underset{t \rightarrow \infty}{\longrightarrow} \sqrt{\frac{2}{\pi}} c \mathbf{E}[|N|]=\frac{2}{\pi} c .
$$

which is precisely (19).

It is now elementary to deduce from (25) that, for $\eta>0$,

$$
\mathbf{E}\left[\left(\ln T_{c}^{\theta}\right)_{+}^{\eta}\right]<\infty \quad \Leftrightarrow \quad 0<\eta<1,
$$

since $(25)$ is equivalent to

$$
u \mathbf{P}\left(\ln T_{c}^{\theta}>u\right) \underset{u \rightarrow \infty}{\longrightarrow} \frac{4 c}{\pi} .
$$

Consequently, Fubini's theorem yields

$$
\mathbf{E}\left[\left(\ln T_{c}^{\theta}\right)_{+}^{\eta}\right]=\int_{0}^{\infty} d u \eta u^{\eta-1} \mathbf{P}\left(\ln T_{c}^{\theta}>u\right),
$$

and by (26) this is finite if and only if

$$
\int^{\infty} d u u^{\eta-2}<\infty \quad \Leftrightarrow \quad \eta<1 .
$$

So, $\mathbf{E}\left[\left(\ln T_{c}^{\theta}\right)_{+}^{\eta}\right]<\infty$ if and only if $0<\eta<1$. Proposition 2.5 is proved.

Now we give several examples of random times $T: C\left(\mathbf{R}_{+}, \mathbf{R}\right) \rightarrow \mathbf{R}_{+}$ which can be studied quite similarly to $T_{c}^{\theta}$. For such times $T$, it will always 
be true that: $H_{T(\theta)}=T(\gamma)$ is equivalent to $T(\theta)=A_{T(\gamma)}$, defined with respect to $Z$, issued from $x_{0} \neq 0$. Using Bougerol's identity, we obtain

$$
\operatorname{sh} \beta_{T(\gamma)} \stackrel{\text { law }}{=} \hat{\beta}_{A_{T(\gamma)}}=\hat{\beta}_{T(\theta)},
$$

where $\left(\hat{\beta}_{u}, u \geqslant 0\right)$ is a one-dimensional Brownian motion independent of $(\beta, \gamma)$ (or equivalently, of $Z$ ). Consequently, denoting by $h_{T}$ the density of $\beta_{T(\gamma)}$, we deduce from $(27)$ that

$$
\mathbf{E}\left[\frac{1}{\sqrt{2 \pi T(\theta)}} \exp \left(-\frac{x^{2}}{2 T(\theta)}\right)\right]=\frac{1}{\sqrt{1+x^{2}}} h_{T}\left(\ln \left(x+\sqrt{1+x^{2}}\right)\right),
$$

or equivalently, changing $x$ in $\sqrt{x}$, we obtain

$$
\mathbf{E}\left[\frac{1}{\sqrt{2 \pi T(\theta)}} \exp \left(-\frac{x}{2 T(\theta)}\right)\right]=\frac{1}{\sqrt{1+x}} h_{T}(\ln (\sqrt{x}+\sqrt{1+x})) .
$$

In a number of cases, $h_{T}$ is known explicitly. We give two examples.

(i) First, we study the exit time from a cone for the planar Brownian motion, and we have

$$
T(\gamma)=T_{-d, c}^{\gamma} \Leftrightarrow T(\theta)=\int_{0}^{T_{-d, c}^{\gamma}} d s \exp \left(2 \beta_{s}\right)=T_{-d, c}^{\theta} .
$$

Thus

$$
\mathbf{E}\left[\frac{1}{\sqrt{2 \pi T_{-d, c}^{\theta}}} \exp \left(-\frac{x}{2 T_{-d, c}^{\theta}}\right)\right]=\frac{1}{\sqrt{1+x}} h_{-d, c}(\ln (\sqrt{x}+\sqrt{1+x})),
$$

where $h_{-d, c}$ is the density of the variable $\beta_{T_{-d, c}^{\gamma}}$. The law of $\beta_{T_{-d, c}^{\gamma}}$ can be obtained from its characteristic function which is given by [31, p. 73]:

$$
\mathbf{E}\left[\exp \left(i \lambda \beta_{T_{-d, c}^{\gamma}}\right)\right]=\mathbf{E}\left[\exp \left(-\frac{\lambda^{2}}{2} T_{-d, c}^{\gamma}\right)\right]=\frac{\operatorname{ch}(\lambda(c-d) / 2)}{\operatorname{ch}(\lambda(c+d) / 2)} .
$$

In particular, for $c=d$, we recover the very classical formula:

$$
\mathbf{E}\left[\exp \left(i \lambda \beta_{T_{-c, c}^{\gamma}}\right)\right]=\frac{1}{\operatorname{ch}(\lambda c)}
$$

It is well known that (see [23], [6])

$$
\begin{aligned}
\mathbf{E}\left[\exp \left(i \lambda \beta_{T_{-c, c}^{\gamma}}\right)\right] & =\frac{1}{\operatorname{ch}(\lambda c)}=\frac{1}{\operatorname{ch}(\pi(\lambda c / \pi))} \\
& =\int_{-\infty}^{\infty} e^{i(\lambda c / \pi) x} \frac{1}{2 \pi} \frac{1}{\operatorname{ch}(x / 2)} d x \\
y=\underline{c x} / \pi & \int_{-\infty}^{\infty} e^{i \lambda y} \frac{1}{2 \pi} \frac{\pi / c}{\operatorname{ch}(y \pi /(2 c))} d y \\
& =\int_{-\infty}^{\infty} e^{i \lambda y} \frac{1}{2 c} \frac{1}{\operatorname{ch}(y \pi /(2 c))} d y .
\end{aligned}
$$


Hence, the density of $\beta_{T_{-c, c}^{\gamma}}$ is

$$
h_{-c, c}(x)=\frac{1}{2 c} \frac{1}{\operatorname{ch}(x \pi /(2 c))}=\frac{1}{c} \frac{1}{e^{x \pi /(2 c)}+e^{-x \pi /(2 c)}},
$$

and

$$
h_{-c, c}(\ln (\sqrt{x}+\sqrt{1+x}))=\frac{1}{c} \frac{1}{(\sqrt{x}+\sqrt{1+x})^{\zeta}+(\sqrt{x}+\sqrt{1+x})^{-\zeta}},
$$

where $\zeta=\pi /(2 c)$. However using the identity

$$
(\sqrt{x}+\sqrt{1+x})^{-\zeta}=(\sqrt{1+x}-\sqrt{x})^{\zeta},
$$

we obtain

$$
h_{-c, c}(\ln (\sqrt{x}+\sqrt{1+x}))=\frac{1}{c} \frac{1}{(\sqrt{x}+\sqrt{1+x})^{\zeta}+(\sqrt{1+x}-\sqrt{x})^{\zeta}} .
$$

So we deduce that (for $c=d$ )

$$
\begin{aligned}
& \mathbf{E}\left[\frac{1}{\sqrt{2 \pi T_{-c, c}^{\theta}}} \exp \left(-\frac{x}{2 T_{-c, c}^{\theta}}\right)\right] \\
& \quad=\frac{1}{c} \frac{1}{\sqrt{1+x}} \frac{1}{(\sqrt{x}+\sqrt{1+x})^{\zeta}+(\sqrt{1+x}-\sqrt{x})^{\zeta}} .
\end{aligned}
$$

(ii) As a second example of a random time $T$, let us consider the time introduced in [33], [10, Exercise 6.2, p. 178] (we use a slightly different notation). Let $\left(\beta_{t}, t \geqslant 0\right)$ be a real-valued Brownian motion and define, for $c>0$ :

$$
\begin{aligned}
& T(\theta) \equiv T_{c}^{\hat{\theta}}=\inf \left\{t: \sup _{s \leqslant t} \theta_{s}-\inf _{s \leqslant t} \theta_{s}=c\right\} \\
& T(\gamma) \equiv T_{c}^{\hat{\gamma}}=\inf \left\{t: \sup _{s \leqslant t} \gamma_{s}-\inf _{s \leqslant t} \gamma_{s}=c\right\} .
\end{aligned}
$$

Thus, from the skew-product representation (1), $\theta_{u} \equiv \gamma_{H_{u}}$, by replacing $u=T_{c}^{\hat{\theta}}$, we obtain

$$
H_{T_{c}^{\hat{\theta}}}=T_{c}^{\hat{\gamma}} \Rightarrow T_{c}^{\hat{\theta}}=\int_{0}^{T_{c}^{\hat{\gamma}}} d s \exp \left(2 \beta_{s}\right) \equiv A_{T_{c}^{\hat{\gamma}}} \cdot
$$

Thus,

$$
\mathbf{E}\left[\frac{1}{\sqrt{2 \pi T_{c}^{\hat{\theta}}}} \exp \left(-\frac{x}{2 T_{c}^{\hat{\theta}}}\right)\right]=\frac{1}{\sqrt{1+x}} h_{c}(\ln (\sqrt{x}+\sqrt{1+x})),
$$


where $h_{c}$ is the density of the variable $\beta_{T_{c}^{\hat{\gamma}}}$. The law of $\beta_{T_{c}^{\hat{\gamma}}}$ can be obtained from its characteristic function which is given by (see [6], [10])

$$
\begin{aligned}
& \mathbf{E}\left[\exp \left(i \lambda \beta_{T_{c}^{\hat{\gamma}}}\right)\right]=\mathbf{E}\left[\exp \left(-\frac{\lambda^{2}}{2} T_{c}^{\hat{\gamma}}\right)\right] \\
& =\frac{1}{(\operatorname{ch}(\lambda c / 2))^{2}}=\frac{1}{(\operatorname{ch}(\pi \lambda c /(2 \pi)))^{2}}=\int_{-\infty}^{\infty} e^{i(\lambda c /(2 \pi)) x} \frac{1}{2 \pi} \frac{x}{\operatorname{sh}(x / 2)} d x \\
& \stackrel{y=c x /(2 \pi)}{=} \int_{-\infty}^{\infty} e^{i \lambda y} \frac{1}{2 \pi} \frac{2 \pi y / c}{\operatorname{sh}(\pi y / c)} \frac{2 \pi}{c} d y=\int_{-\infty}^{\infty} e^{i \lambda y} \frac{2 \pi}{c^{2}} \frac{y}{\operatorname{sh}(\pi y / c)} d y .
\end{aligned}
$$

So, the density of $\beta_{T_{c}^{\hat{\gamma}}}$ is

$$
h_{c}(y)=\frac{2 \pi y}{c^{2}} \frac{1}{\operatorname{sh}(\pi y / c)}=\frac{4 \pi}{c^{2}} \frac{y}{e^{\pi y / c}-e^{-\pi y / c}},
$$

and

$$
h_{c}(\ln (\sqrt{x}+\sqrt{1+x}))=\frac{4 \pi}{c^{2}} \frac{\ln (\sqrt{x}+\sqrt{1+x})}{(\sqrt{x}+\sqrt{1+x})^{\hat{\zeta}}-(\sqrt{x}+\sqrt{1+x})^{-\hat{\zeta}}},
$$

where $\hat{\zeta}=\pi / c$. Thus

$$
\begin{aligned}
& \mathbf{E}\left[\frac{1}{\sqrt{2 \pi T_{c}^{\hat{\theta}}}} \exp \left(-\frac{x}{2 T_{c}^{\hat{\theta}}}\right)\right] \\
& =\frac{4 \pi}{c^{2}} \frac{1}{\sqrt{1+x}} \frac{\ln (\sqrt{x}+\sqrt{1+x})}{(\sqrt{x}+\sqrt{1+x})^{\hat{\zeta}}-(\sqrt{1+x}-\sqrt{x})^{\hat{\zeta}}} .
\end{aligned}
$$

We note that this study can be related to [30]; and, more precisely, $\beta_{T_{c}^{\hat{\gamma}}}$ and $T_{c}^{\hat{\gamma}}$ correspond to the variables $C_{2}$ and $\widehat{C_{2}}$, respectively (see, e.g., [30, Table 6, p. 312]).

Let us now return to the case of $T_{-c, c}^{\theta}$ (example (i)). More specifically, we shall obtain its density function $f(t)$.

Proposition 2.6. The density function $f$ of $T_{-c, c}^{\theta}$ is given by

$$
f(t)=\frac{1}{\sqrt{2} c} \sum_{k=0}^{\infty}(-1)^{k} \frac{\Gamma\left(\nu_{k}\right)}{\Gamma\left(2 \nu_{k}\right)} \frac{1}{\sqrt{t}} e^{-1 /(4 t)} M_{1 / 2, \nu_{k}}\left(\frac{1}{2 t}\right),
$$

where $M_{a, b}(\cdot)$ is the Whittaker function with parameters $a, b$. Equivalently,

$$
\begin{aligned}
f(t)= & \frac{\sqrt{2}}{c} \sum_{k=0}^{\infty}(-1)^{k} \frac{1}{\sqrt{t}} e^{-1 /(2 t)}\left(\frac{1}{2 t}\right)^{\nu_{k}+1 / 2} \nu_{k} \\
& \times \sum_{n=0}^{\infty} \frac{\Gamma\left(\nu_{k}+n\right)}{\Gamma\left(2 \nu_{k}+n+1\right)} \frac{1}{n !}\left(\frac{1}{2 t}\right)^{n},
\end{aligned}
$$

where $\nu_{k}=\pi(2 k+1) /(4 c)$. 
P r o o f. The following calculation relies upon [11]. We denote

$$
\varphi_{\zeta}(x)=(\sqrt{x}+\sqrt{1+x})^{\zeta}+(\sqrt{1+x}-\sqrt{x})^{\zeta} .
$$

Noting that

$$
\sqrt{1+x}=\operatorname{ch} \frac{y}{2} \quad \Longleftrightarrow \quad y=2 \arg \operatorname{ch}(\sqrt{1+x})
$$

we get

$$
\varphi_{\zeta}(x)=\left(\operatorname{sh} \frac{y}{2}+\operatorname{ch} \frac{y}{2}\right)^{\zeta}+\left(\operatorname{ch} \frac{y}{2}-\operatorname{sh} \frac{y}{2}\right)^{\zeta}=2 \operatorname{ch} \frac{y \zeta}{2} .
$$

Thus, from (34), we have

$$
I I \equiv \mathbf{E}\left[\frac{1}{\sqrt{2 \pi T_{-c, c}^{\theta}}} \exp \left(-\frac{x}{2 T_{-c, c}^{\theta}}\right)\right]=\frac{1}{\psi} \frac{1}{\operatorname{ch}(y / 2)} \frac{1}{\operatorname{ch}(\pi y /(2 \psi))},
$$

where $\psi=2 c$. However, expanding $\operatorname{ch}(\pi y /(2 \psi))$, we get

$$
\frac{1}{\operatorname{ch}(\pi y /(2 \psi))}=2 \frac{e^{-\pi y /(2 \psi)}}{1+e^{-\pi y / \psi}}=2 \sum_{k=0}^{\infty}\left(-e^{-\pi y / \psi}\right)^{k} e^{-\pi y /(2 \psi)},
$$

and from (41), we deduce that

$$
\begin{aligned}
I I & =\sum_{k=0}^{\infty} \frac{2}{\psi} \frac{(-1)^{k}}{\operatorname{ch}(y / 2)} e^{-\pi(2 k+1) y /(2 \psi)} \\
& =\sum_{k=0}^{\infty} \frac{4(-1)^{k}}{\psi \sqrt{2} \sqrt{2 \operatorname{sh}(y / 2) \operatorname{ch}(y / 2)}} \sqrt{\frac{\operatorname{sh}(y / 2)}{\operatorname{ch}(y / 2)}} e^{-\nu_{k} y} \\
& =\sum_{k=0}^{\infty} \frac{4(-1)^{k}}{\psi \sqrt{2} \sqrt{2 \operatorname{sh}(y / 2) \operatorname{ch}(y / 2)}} \sqrt{\operatorname{th} \frac{y}{2}} e^{-\nu_{k} y},
\end{aligned}
$$

where $\nu_{k}=\pi(2 k+1) /(2 \psi)$.

From (40), we have that $1+x=\operatorname{ch}^{2}(y / 2)$ if and only if $x=\operatorname{sh}^{2}(y / 2)$, thus

$$
\left(\operatorname{th} \frac{y}{2}\right)^{1 / 2}=\sqrt{\frac{\operatorname{sh}(y / 2)}{\operatorname{ch}(y / 2)}}=\left(\frac{\sqrt{x}}{\sqrt{1+x}}\right)^{1 / 2}=\left(\frac{x}{1+x}\right)^{1 / 4}
$$

Moreover, we know that (see [1, equation 8.6.10] or [22])

$$
i \sqrt{\frac{\pi}{2 \operatorname{sh} y}} e^{-\nu_{k} y}=Q_{\nu_{k}-1 / 2}^{1 / 2}(\operatorname{ch} y)
$$


where $\left\{Q_{b}^{a}(\cdot)\right\}$ is the family of Legendre functions and ch $y=2 x+1$. So, we deduce

$$
I I=\sum_{k=0}^{\infty} \frac{4(-i)}{\psi \sqrt{\pi}}(-1)^{k}\left(\frac{x}{1+x}\right)^{1 / 4} Q_{\nu_{k}-1 / 2}^{1 / 2}(2 x+1) .
$$

By using formula (7.621.9) in [17]

$$
\int_{0}^{\infty} e^{-s w} M_{l, \nu_{k}}(w) \frac{d w}{w}=\frac{2 \Gamma\left(1+2 \nu_{k}\right) e^{-i \pi l}}{\Gamma\left(1 / 2+\nu_{k}+l\right)}\left(\frac{s-1 / 2}{s+1 / 2}\right)^{l / 2} Q_{\nu_{k}-1 / 2}^{l}(2 s),
$$

with $l=1 / 2, \nu_{k}=\pi(2 k+1) /(2 \psi), s=x+1 / 2$ and $M_{., .}(\cdot)$ denoting the Whittaker function, which is defined as

$$
M_{a, b}(w)=w^{b+1 / 2} e^{-w / 2} \frac{\Gamma(2 b+1)}{\Gamma(1 / 2+b-a)} \sum_{n=0}^{\infty} \frac{\Gamma(1 / 2+b-a+n)}{\Gamma(2 b+1+n)} \frac{w^{n}}{n !},
$$

we have

$-2 i \frac{\Gamma\left(1+2 \nu_{k}\right)}{\Gamma\left(1+\nu_{k}\right)}\left(\frac{x}{1+x}\right)^{1 / 4} Q_{\nu_{k}-1 / 2}^{1 / 2}(2 x+1)=\int_{0}^{\infty} e^{-s w} M_{1 / 2, \nu_{k}}(w) \frac{d w}{w}$.

From (42) and by changing the variable $w=1 /(2 t)$, we deduce

$$
\begin{aligned}
I I & =\sum_{k=0}^{\infty} \frac{2}{\psi \sqrt{\pi}}(-1)^{k} \frac{\Gamma\left(\nu_{k}+1\right)}{\Gamma\left(2 \nu_{k}+1\right)} \int_{0}^{\infty} \frac{d w}{w} \exp \left(-w\left(x+\frac{1}{2}\right)\right) M_{1 / 2, \nu_{k}}(w) \\
& =\sum_{k=0}^{\infty} \int_{0}^{\infty} \frac{d t}{t} \frac{2}{\psi \sqrt{\pi}}(-1)^{k} \frac{\Gamma\left(\nu_{k}+1\right)}{\Gamma\left(2 \nu_{k}+1\right)} \exp \left(-\frac{1}{4 t}-\frac{x}{2 t}\right) M_{1 / 2, \nu_{k}}\left(\frac{1}{2 t}\right) .
\end{aligned}
$$

By using the equations (41) and (45), we conclude that

$$
\begin{aligned}
\mathbf{E} & {\left[\frac{1}{\sqrt{2 \pi T_{-c, c}^{\theta}}} \exp \left(-\frac{x}{2 T_{-c, c}^{\theta}}\right)\right] } \\
= & \sum_{k=0}^{\infty} \int_{0}^{\infty} \frac{d t}{t} \frac{2}{\psi \sqrt{\pi}}(-1)^{k} \frac{\Gamma\left(\nu_{k}+1\right)}{\Gamma\left(2 \nu_{k}+1\right)} \exp \left(-\frac{1}{4 t}-\frac{x}{2 t}\right) M_{1 / 2, \nu_{k}}\left(\frac{1}{2 t}\right) \\
= & \sum_{k=0}^{\infty} \int_{0}^{\infty} \frac{d t}{t} \frac{2}{\psi \sqrt{\pi}}(-1)^{k} \frac{\Gamma\left(\pi(4 c)^{-1}(2 k+1)+1\right)}{\Gamma\left(2 \pi(4 c)^{-1}(2 k+1)+1\right)} \\
& \times \exp \left(-\frac{\pi}{4 t}-\frac{x}{2 t}\right) M_{1 / 2, \pi(4 c)^{-1}(2 k+1)}\left(\frac{1}{2 t}\right) .
\end{aligned}
$$

Thus, the density function $f$ of $T_{-c, c}^{\theta}$ is given by

$$
f(t)=\frac{2 \sqrt{2}}{\psi} \sum_{k=0}^{\infty}(-1)^{k} \frac{\Gamma\left(\nu_{k}+1\right)}{\Gamma\left(2 \nu_{k}+1\right)} \frac{1}{\sqrt{t}} e^{-1 /(4 t)} M_{1 / 2, \nu_{k}}\left(\frac{1}{2 t}\right)
$$




$$
\begin{gathered}
=\frac{\sqrt{2}}{c} \sum_{k=0}^{\infty}(-1)^{k} \frac{\Gamma\left(\pi(4 a)^{-1}(2 k+1)+1\right)}{\Gamma(\pi /(2 a)(2 k+1)+1)} \frac{1}{\sqrt{t}} e^{-1 /(4 t)} \\
\times M_{1 / 2, \pi(4 a)^{-1}(2 k+1)}\left(\frac{1}{2 t}\right) \\
=\frac{\sqrt{2}}{c} \sum_{k=0}^{\infty}(-1)^{k} \frac{\nu_{k} \Gamma\left(\nu_{k}\right)}{2 \nu_{k} \Gamma\left(2 \nu_{k}\right)} \frac{1}{\sqrt{t}} e^{-1 /(4 t)} M_{\frac{1}{2}, \nu_{k}}\left(\frac{1}{2 t}\right),
\end{gathered}
$$

where the Whittaker function $M_{1 / 2, \nu_{k}}(1 /(2 t))$ is

$$
\begin{aligned}
& M_{1 / 2, \pi(4 c)^{-1}(2 k+1)}\left(\frac{1}{2 t}\right)=\left(\frac{1}{2 t}\right)^{\pi(4 c)^{-1}(2 k+1)+1 / 2} \\
& \quad \times e^{-1 /(4 t)} \frac{\Gamma\left(\pi(2 c)^{-1}(2 k+1)+1\right)}{\Gamma\left(\pi(4 c)^{-1}(2 k+1)\right)} \\
& \quad \times \sum_{n=0}^{\infty} \frac{\Gamma\left(\pi(4 c)^{-1}(2 k+1)+n\right)}{\Gamma\left(\pi(2 c)^{-1}(2 k+1)+1+n\right)} \frac{1}{n !}\left(\frac{1}{2 t}\right)^{n} \\
& =\left(\frac{1}{2 t}\right)^{\nu_{k}+1 / 2} e^{-1 /(4 t)} \frac{\Gamma\left(2 \nu_{k}+1\right)}{\Gamma\left(\nu_{k}\right)} \sum_{n=0}^{\infty} \frac{\Gamma\left(\nu_{k}+n\right)}{\Gamma\left(2 \nu_{k}+1+n\right)} \frac{1}{n !}\left(\frac{1}{2 t}\right)^{n} \\
& =\left(\frac{1}{2 t}\right)^{\nu_{k}+1 / 2} e^{-1 /(4 t)} \cdot 2 \nu_{k} \frac{\Gamma\left(2 \nu_{k}\right)}{\Gamma\left(\nu_{k}\right)} \sum_{n=0}^{\infty} \frac{\Gamma\left(\nu_{k}+n\right)}{\left(2 \nu_{k}+n\right) \Gamma\left(2 \nu_{k}+n\right)} \frac{1}{n !}\left(\frac{1}{2 t}\right)^{n} .
\end{aligned}
$$

Thus, from (47) and (48), we deduce (39). Proposition 2.6 is proved.

Next, we present the graphs of different approximations $f_{K, N}(t)$ of $f(t)$, in (39), where $f_{K, N}$ denotes the sum in the series in (39) of the terms for $k \leqslant K$, and $n \leqslant N$.

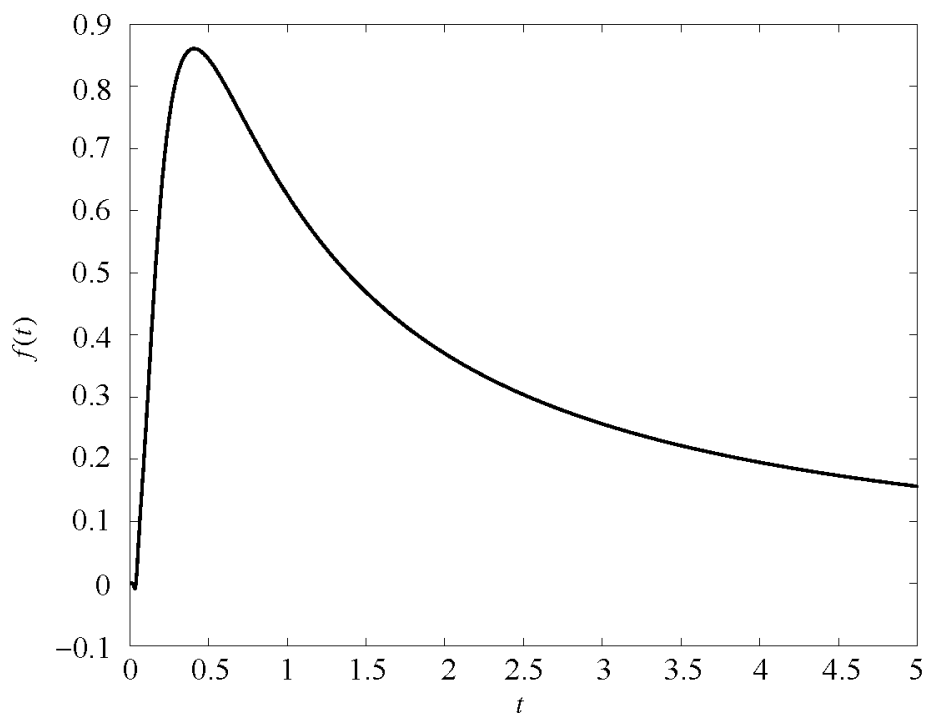

Fig. 3. Graph of $f_{9,9}(t)$, with $c=2 \pi$. 


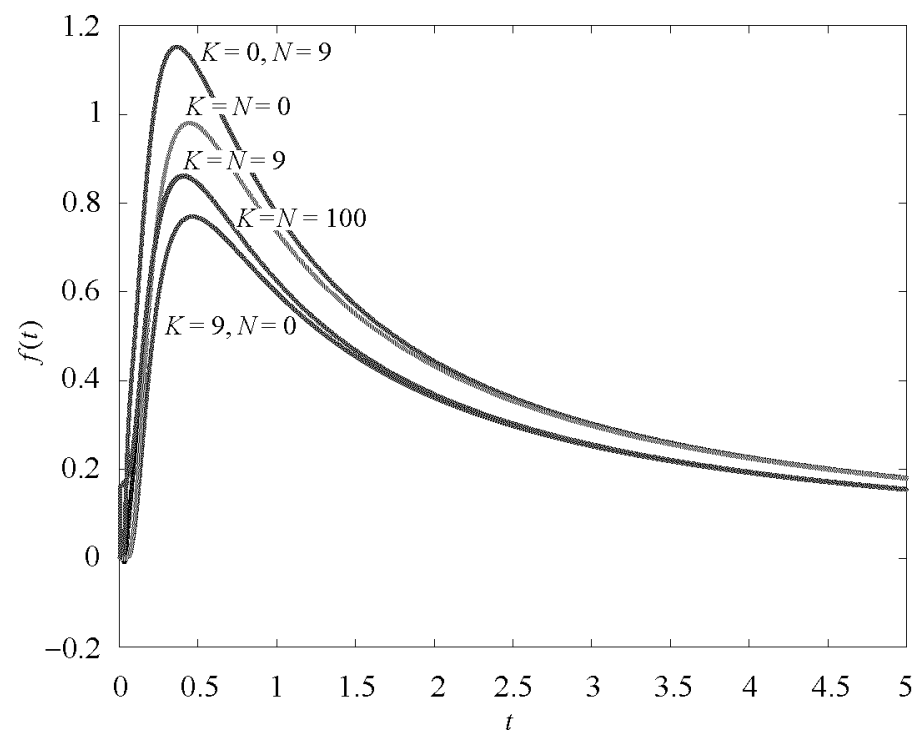

Fig. 4. Graph of $f_{K, N}(t)$ for several values of $K$ and $N$, with $c=2 \pi$.

$\mathrm{R}$ e $\mathrm{m}$ a r k s. (i) Figure 3 represents the approximation of the density function $f$ with respect to the time $t$ (for $K$ and $N \leqslant 9$ ), with $c=2 \pi$, whereas Figure 4 represents the approximation of $f$ with respect to the time $t$ for several values of $k$ and $n$, with $c=2 \pi$.

(ii) From Figure 4, we can remark that the approximation $K$ and $N \leqslant 9$ is sufficiently good (comparing to the one for $K$ and $N \leqslant 100$ ).

(iii) For the case $K$ and $N \leqslant 9$ it seems that locally, in a small area around $0, f(t)<0$ which is not right. This is due to the first negative $(k=1)$ term of the sum and to the fact that we have omitted many terms. However, this is not a problem because it appears only locally. Similar irregularities have already been observed in previous articles [18, p. 275].

2.6. On the first moment of $\ln T_{-c, c}^{\theta}$. This subsection is related to a result in [11].

Proposition 2.7. The first moment of $\ln T_{-c, c}^{\theta}$ has the following integral representation:

$$
\mathbf{E}\left[\ln T_{-c, c}^{\theta}\right]=2 \int_{0}^{\infty} \frac{d z}{\operatorname{ch}(\pi z / 2)} \ln \operatorname{sh}(c z)+\ln 2+c_{E},
$$

where $c_{E}=-\Gamma^{\prime}(1)$ is the Euler-Mascheroni constant (also called Euler's constant).

P r o o f. Let us return to equations (2) and (6). So, for $t=T_{-c, c}^{\theta}$, we have

$$
\theta_{T_{-c, c}^{\theta}}=\gamma_{H_{T_{-c, c}^{\theta}}} \Longleftrightarrow H_{T_{-c, c}^{\theta}}=T_{-c, c}^{\gamma} \quad \Longleftrightarrow \quad T_{-c, c}^{\theta}=A_{T_{-c, c}^{\gamma}} .
$$


Thus, for $\varepsilon>0$

$$
\mathbf{E}\left[\left(T_{-c, c}^{\theta}\right)^{\varepsilon}\right]=\mathbf{E}\left[\left(A_{T_{-c, c}^{\gamma}}\right)^{\varepsilon}\right] .
$$

Consider $\left(\delta_{u}, u \geqslant 0\right)$ a Brownian motion independent of $A_{t}$. Then, Bougerol's identity and the scaling property yield $\left(\mathscr{G}_{a}\right.$ denotes a gamma variable with parameter $a$, and $N^{2} \stackrel{\text { law }}{=} 2 \mathscr{G}_{1 / 2}$ )

$$
\begin{aligned}
\mathbf{E}\left[\left(\operatorname{sh} B_{t}\right)^{2 \varepsilon}\right] & =\mathbf{E}\left[\left(\delta_{A_{t}}\right)^{2 \varepsilon}\right]=\mathbf{E}\left[A_{t}^{\varepsilon}\left(\delta_{1}\right)^{2 \varepsilon}\right]=\mathbf{E}\left[A_{t}^{\varepsilon}\right] \mathbf{E}\left[\left(2 \mathscr{G}_{1 / 2}\right)^{\varepsilon}\right] \\
& =\mathbf{E}\left[A_{t}^{\varepsilon}\right] \cdot 2^{\varepsilon} \frac{\Gamma(1 / 2+\varepsilon)}{\Gamma(1 / 2)}
\end{aligned}
$$

because

$$
\mathbf{E}\left[\left(\mathscr{G}_{1 / 2}\right)^{\varepsilon}\right]=\int_{0}^{\infty} x^{\varepsilon+1 / 2-1} \frac{e^{-x}}{\Gamma(1 / 2)} d x=\frac{\Gamma(1 / 2+\varepsilon)}{\Gamma(1 / 2)} .
$$

Thus, for $t=T_{-c, c}^{\gamma}$, we have

$$
\mathbf{E}\left[\left(\operatorname{sh} B_{T_{-c, c}^{\gamma}}\right)^{2 \varepsilon}\right]=\mathbf{E}\left[A_{T_{-c, c}^{\gamma}}^{\varepsilon}\right] \cdot 2^{\varepsilon} \frac{\Gamma(1 / 2+\varepsilon)}{\Gamma(1 / 2)} .
$$

Recall that [23], [6]

$$
\mathbf{E}\left[\exp \left(i \lambda B_{T_{-c, c}^{\gamma}}\right)\right]=\mathbf{E}\left[\exp \left(-\frac{\lambda^{2}}{2} T_{-c, c}^{\gamma}\right)\right]=\frac{1}{\operatorname{ch}(\lambda c)},
$$

and the density of $\beta_{T_{-c, c}^{\gamma}}$ is

$$
h_{-c, c}(y)=\frac{1}{2 c} \frac{1}{\operatorname{ch}(y \pi /(2 c))}=\frac{1}{c} \frac{1}{e^{y \pi /(2 c)}+e^{-y \pi /(2 c)}} .
$$

Thus, on the left-hand side of (51), we have:

$$
\begin{aligned}
\mathbf{E}\left[\left(\operatorname{sh} B_{T_{-c, c}^{\gamma}}\right)^{2 \varepsilon}\right] & =\int_{-\infty}^{\infty} \frac{d y}{2 c} \frac{1}{\operatorname{ch}(\pi y /(2 c))}(\operatorname{sh} y)^{2 \varepsilon} \\
& =\int_{0}^{\infty} \frac{d y}{c} \frac{1}{\operatorname{ch}(\pi y /(2 c))}(\operatorname{sh} y)^{2 \varepsilon} \\
& =\int_{0}^{\infty} d z \frac{1}{\operatorname{ch}(\pi z / 2)}(\operatorname{sh}(c z))^{2 \varepsilon}
\end{aligned}
$$

where we have made the change of variable $z=y / c$. Hence, from (51), by writing

$$
\mathbf{E}\left[A_{T_{-c, c}^{\gamma}}^{\varepsilon}\right]=\mathbf{E}\left[\left(T_{-c, c}^{\theta}\right)^{\varepsilon}\right]=\mathbf{E}\left[e^{\varepsilon \ln T_{-c, c}^{\theta}}\right],
$$


we deduce

$$
\frac{\Gamma(1 / 2+\varepsilon)}{\Gamma(1 / 2)} \mathbf{E}\left[e^{\varepsilon \ln T_{-c, c}^{\theta}}\right]=\frac{1}{2^{\varepsilon}} \int_{0}^{\infty} \frac{d z}{\operatorname{ch}(\pi z / 2)}(\operatorname{sh}(c z))^{2 \varepsilon},
$$

and by removing 1 from both sides, we obtain

$$
\frac{\Gamma(1 / 2+\varepsilon)}{\Gamma(1 / 2)} \mathbf{E}\left[e^{\left.\varepsilon \ln T_{-c, c}^{\theta}\right]}-1=\int_{0}^{\infty} \frac{d z}{\operatorname{ch}(\pi z / 2)}\left(\frac{(\operatorname{sh}(c z))^{2 \varepsilon}}{2^{\varepsilon}}-1\right) .\right.
$$

On the left-hand side, we apply the trivial identity $a b-1=a(b-1)+a-1$ with $a=\Gamma(1 / 2+\varepsilon) / \Gamma(1 / 2)$ and $b=\mathbf{E}\left[e^{\varepsilon \ln T_{-c, c}^{\theta}}\right]$, we divide by $\varepsilon$ and we take the limit as $\varepsilon \rightarrow 0$. Thus,

$$
\frac{a(b-1)}{\varepsilon}=\frac{\Gamma(1 / 2+\varepsilon)}{\Gamma(1 / 2)} \frac{\mathbf{E}\left[e^{\left.\varepsilon \ln T_{-c, c}^{\theta}\right]}-1\right.}{\varepsilon} \underset{\varepsilon \rightarrow 0}{\longrightarrow} \mathbf{E}\left[\ln T_{-c, c}^{\theta}\right],
$$

and

$$
\begin{aligned}
& \frac{a-1}{\varepsilon}=\frac{1}{\varepsilon}\left(\frac{\Gamma(1 / 2+\varepsilon)}{\Gamma(1 / 2)}-1\right)=\frac{1}{\Gamma(1 / 2)}\left(\frac{\Gamma(1 / 2+\varepsilon)-\Gamma(1 / 2)}{\varepsilon}\right) \\
& \underset{\varepsilon \rightarrow 0}{\longrightarrow} \frac{1}{\sqrt{\pi}} \Gamma^{\prime}\left(\frac{1}{2}\right)=\frac{1}{\sqrt{\pi}}(-\sqrt{\pi})\left(c_{E}+2 \ln 2\right)=-\left(c_{E}+2 \ln 2\right) .
\end{aligned}
$$

On the right-hand side of (52), we have

$$
\frac{1}{\varepsilon}\left[\left(\frac{(\operatorname{sh}(c z))^{2}}{2}\right)^{\varepsilon}-1\right]=\frac{1}{\varepsilon}\left[\exp \left(\varepsilon \ln \frac{(\operatorname{sh}(c z))^{2}}{2}\right)-1\right],
$$

hence

$$
\begin{aligned}
\frac{1}{\varepsilon} \int_{0}^{\infty} \frac{d z}{\operatorname{ch}(\pi z / 2)}\left(\frac{(\operatorname{sh}(c z))^{2 \varepsilon}}{2^{\varepsilon}}-1\right) & \underset{\varepsilon \rightarrow 0}{\longrightarrow} \int_{0}^{\infty} \frac{d z}{\operatorname{ch}(\pi z / 2)} \ln \frac{(\operatorname{sh}(c z))^{2}}{2} \\
& =-\ln 2+2 \int_{0}^{\infty} \frac{d z}{\operatorname{ch}(\pi z / 2)} \ln \operatorname{sh}(c z)
\end{aligned}
$$

which finishes the proof.

$\mathrm{R}$ e m a r k. a) We denote now

$$
F(c) \equiv \int_{0}^{\infty} \frac{d z}{\operatorname{ch}(\pi z / 2)} \ln \operatorname{sh}(c z)
$$

Thus,

$$
F(c)-\ln c \equiv \int_{0}^{\infty} \frac{d z}{\operatorname{ch}(\pi z / 2)} \ln \frac{\operatorname{sh}(c z)}{c} \stackrel{c \rightarrow 0}{\longrightarrow} \int_{0}^{\infty} \frac{d z \ln z}{\operatorname{ch}(\pi z / 2)} \approx-0.7832 .
$$

b) More generally, we denote

$$
F(c, \delta) \equiv \int_{0}^{\infty} \frac{d z}{\operatorname{ch}(\delta z)} \ln \operatorname{sh}(c z),
$$

and, changing the variables: $z=\frac{\pi}{2 \delta} u$, we obtain

$$
F(c, \delta)=\frac{\pi}{2 \delta} \int_{0}^{\infty} \frac{d u}{\operatorname{ch}(\pi / 2 u)} \ln \operatorname{sh} \frac{c \pi u}{2 \delta}=\frac{\pi}{2 \delta} F\left(c \frac{\pi}{2 \delta}\right) .
$$


3. The Ornstein-Uhlenbeck case.

3.1. An identity in law for Ornstein-Uhlenbeck processes, which is connected to Bougerol's identity. Consider the complexvalued Ornstein-Uhlenbeck process

$$
Z_{t}=z_{0}+\widetilde{Z}_{t}-\lambda \int_{0}^{t} Z_{s} d s
$$

where $\widetilde{Z}_{t}$ is a complex-valued Brownian motion, $z_{0} \in \mathbf{C}$ and $\lambda \geqslant 0$, and let $T_{c}^{(\lambda)} \equiv T_{-c, c}^{\theta^{Z}} \equiv \inf \left\{t \geqslant 0:\left|\theta_{t}^{Z}\right|=c\right\}\left(\theta_{t}^{Z}\right.$ is the continuous winding process associated to $Z$ ) denote the first hitting time of the symmetric conic boundary of angle $c$ for $Z$. It is well known (see, e.g., [31]) that

$$
Z_{t}=e^{-\lambda t}\left(z_{0}+\int_{0}^{t} e^{\lambda s} d \widetilde{Z}_{s}\right)=e^{-\lambda t} \mathbf{B}_{\alpha_{t}}
$$

where, by Dambis-Dubins-Schwarz theorem, $\left(\mathbf{B}_{t}, t \geqslant 0\right)$ is a complex-valued Brownian motion starting from $z_{0}$ and

$$
\alpha_{t}=\int_{0}^{t} e^{2 \lambda s} d s=\frac{e^{2 \lambda t}-1}{2 \lambda} .
$$

We are interested in the study of the continuous winding process $\theta_{t}^{Z}=$ $\operatorname{Im}\left(\int_{0}^{t} Z_{s}^{-1} d Z_{s}\right), t \geqslant 0$. By applying Itô's formula to (58), we have

$$
d Z_{s}=e^{-\lambda s}(-\lambda) \mathbf{B}_{\alpha_{s}} d s+e^{-\lambda s} d \mathbf{B}_{\alpha_{s}} .
$$

We divide by $Z_{s}$ and we obtain

$$
\frac{d Z_{s}}{Z_{s}}=-\lambda d s+\frac{d \mathbf{B}_{\alpha_{s}}}{\mathbf{B}_{\alpha_{s}}},
$$

hence

$$
\operatorname{Im}\left(\frac{d Z_{s}}{Z_{s}}\right)=\operatorname{Im}\left(\frac{d \mathbf{B}_{\alpha_{s}}}{\mathbf{B}_{\alpha_{s}}}\right)
$$

which means that

$$
\theta_{t}^{Z}=\theta_{\alpha_{t}}^{\mathbf{B}}
$$

Thus, the following proposition holds.

Proposition 3.1. Using the previously introduced notation, we have

$$
\theta_{t}^{Z}=\theta_{\alpha_{t}}^{\mathbf{B}},
$$

and

$$
T_{c}^{(\lambda)}=\frac{1}{2 \lambda} \ln \left(1+2 \lambda T_{-c, c}^{\theta^{\mathrm{B}}}\right),
$$

where $T_{-c, c}^{\theta^{\mathrm{B}}}$ is the exit time from a cone of angle $c$ for the complex-valued Brownian motion $\mathbf{B}$. 
P r o o f. We define

$$
T_{c}^{(\lambda)} \equiv T_{-c, c}^{\theta^{Z}} \equiv \inf \left\{t \geqslant 0:\left|\theta_{t}^{Z}\right|=c\right\}=\inf \left\{t \geqslant 0:\left|\theta_{\alpha_{t}}^{\mathbf{B}}\right|=c\right\} .
$$

Thus, we deduce that $\alpha_{T_{c}^{(\lambda)}}=T_{-c, c}^{\theta^{\mathrm{B}}} \equiv T_{-c, c}^{\theta}$. However, $T_{-c, c}^{\theta}$ (the exit time from a cone for the Brownian motion) has already been studied in the previous section and we know the explicit formula of its density function (Proposition 2.6). Thus

$$
T_{c}^{(\lambda)}=\alpha^{-1}\left(T_{-c, c}^{\theta^{\mathrm{B}}}\right)=\alpha^{-1}\left(T_{-c, c}^{\theta}\right),
$$

where $\alpha^{-1}(t)=(2 \lambda)^{-1} \ln (1+2 \lambda t)$. Consequently,

$$
T_{c}^{(\lambda)}=\frac{1}{2 \lambda} \ln \left(1+2 \lambda T_{-c, c}^{\theta}\right),
$$

and

$$
\mathbf{E}\left[T_{c}^{(\lambda)}\right]=\frac{1}{2 \lambda} \mathbf{E}\left[\ln \left(1+2 \lambda T_{-c, c}^{\theta}\right)\right],
$$

which finishes the proof.

From now on, for simplicity, we shall take $z_{0}=1$ (but this is really no restriction, as the dependency in $z_{0}$, which is exhibited in (7), is very simple). The following proposition may be considered as an extension of the identity in law (ii) in Proposition 2.3, which results from Bougerol's identity.

Proposition 3.2. Consider two independent Ornstein-Uhlenbeck processes $\left(Z_{t}^{\lambda}, t \geqslant 0\right)$ and $\left(U_{t}^{\lambda}, t \geqslant 0\right)$, the first one is complex valued and the second one is real valued, both starting from a point different from 0 and call $T_{b}^{(\lambda)}\left(U^{\lambda}\right)=\inf \left\{t \geqslant 0: e^{\lambda t} U_{t}^{\lambda}=b\right\}$. Then an Ornstein-Uhlenbeck extension of identity in law (ii) in Proposition 2.3 is the following:

$$
\theta_{T_{b}^{(\lambda)}\left(U^{\lambda}\right)}^{Z^{\lambda}} \stackrel{\text { law }}{=} C_{a(b)},
$$

where $a(x)=\arg \operatorname{sh} x$.

P r o o f. Consider the second Ornstein-Uhlenbeck process $\left(U_{t}^{\lambda}, t \geqslant 0\right)$ independent of the first one. Then, taking equation (58) for $U_{t}^{\lambda}$, we have

$$
e^{\lambda t} U_{t}^{\lambda}=\delta_{\left(e^{2 \lambda t}-1\right) /(2 \lambda)},
$$

where $\left(\delta_{t}, t \geqslant 0\right)$ is a complex-valued Brownian motion starting from $z_{0}=1$. Thus

$$
T_{b}^{(\lambda)}\left(U^{\lambda}\right)=\frac{1}{2 \lambda} \ln \left(1+2 \lambda T_{b}^{\delta}\right) .
$$

Equation (59) for $t=(2 \lambda)^{-1} \ln \left(1+2 \lambda T_{b}^{\delta}\right)$ (equivalently, $\alpha(t)=T_{b}^{\delta}$ ) becomes (we suppose that $z_{0}=1$ )

$$
\theta_{T_{b}^{(\lambda)}\left(U^{\lambda}\right)}^{Z^{\lambda}}=\theta_{(2 \lambda)^{-1} \ln \left(1+2 \lambda T_{b}^{\delta}\right)}^{Z^{\lambda}}=\theta_{u=T_{b}^{\delta}}^{\mathbf{B}} \stackrel{\text { law }}{=} C_{a(b)} .
$$


3.2. On the distribution of $T_{-c, c}^{\theta}$ for an Ornstein-Uhlenbeck process. Now we turn to the study of the density function of

$$
T_{c}^{(\lambda)} \equiv T_{-c, c}^{\theta^{Z}} \equiv \inf \left\{t \geqslant 0:\left|\theta_{t}^{Z}\right|=c\right\},
$$

and its first moment.

Proposition 3.3. Asymptotically for $\lambda$ large, for $z_{0}=1$, we have

$$
2 \lambda \mathbf{E}\left[T_{c}^{(\lambda)}\right]-\ln (2 \lambda) \underset{\lambda \rightarrow \infty}{\longrightarrow} \mathbf{E}\left[\ln T_{-c, c}^{\theta}\right]
$$

and

$$
\mathbf{E}\left[\ln T_{-c, c}^{\theta}\right]=2 \int_{0}^{\infty} \frac{d z}{\operatorname{ch}(\pi z / 2)} \ln \operatorname{sh}(c z)+\ln 2+c_{E},
$$

where $c_{E}$ is Euler's constant.

For $c<\pi / 8$, we have the asymptotic equivalence

$$
\frac{1}{\lambda}\left(\mathbf{E}\left[T_{c}^{(\lambda)}\right]-\mathbf{E}\left[\left(\operatorname{sh} B_{T_{-c, c}^{\gamma}}\right)^{2}\right]\right) \underset{\lambda \rightarrow 0}{\longrightarrow}-\frac{1}{3} \mathbf{E}\left[\left(\operatorname{sh} B_{T_{-c, c}^{\gamma}}\right)^{4}\right] .
$$

Equivalently,

$$
\left.\frac{d}{d \lambda}\right|_{\lambda=0} \mathbf{E}\left[T_{c}^{(\lambda)}\right]=\lim _{\lambda \rightarrow 0}\left[\frac{1}{\lambda}\left(\mathbf{E}\left[T_{c}^{(\lambda)}\right]-\mathbf{E}\left[T_{c}^{(0)}\right]\right)\right]=-\frac{1}{3} \mathbf{E}\left[\left(\operatorname{sh} B_{T_{-c, c}^{\gamma}}\right)^{4}\right] .
$$

Moreover,

$$
\mathbf{E}\left[\left(\operatorname{sh} B_{T_{-c, c}^{\gamma}}\right)^{4}\right]=\int_{0}^{\infty} \frac{d z}{\operatorname{ch}(\pi z / 2)}(\operatorname{sh}(c z))^{4} .
$$

More precisely, for $c<\pi / 8$,

$$
\mathbf{E}\left[\left(\operatorname{sh} B_{T_{-c, c}^{\gamma}}\right)^{4}\right]=\frac{1}{8}\left(\frac{1}{\cos (4 c)}-4 \frac{1}{\cos (2 c)}+3\right),
$$

and asymptotically:

$$
\mathbf{E}\left[\left(\operatorname{sh} B_{T_{-c, c}^{\gamma}}\right)^{4}\right] \underset{c \rightarrow 0}{\simeq} 5 c^{4} .
$$

P r o o f. Case 1: $\lambda$ large. Let us return to equation (63). As $\lambda \rightarrow \infty$, we have

$$
\begin{aligned}
\mathbf{E}\left[T_{c}^{(\lambda)}\right] & =\frac{1}{2 \lambda} \mathbf{E}\left[\ln \left(1+2 \lambda T_{-c, c}^{\theta}\right)\right]=\frac{1}{2 \lambda} \mathbf{E}\left[\ln \left(2 \lambda\left(T_{-c, c}^{\theta}+\frac{1}{2 \lambda}\right)\right)\right] \\
& =\frac{\ln (2 \lambda)}{2 \lambda}+\frac{1}{2 \lambda} \mathbf{E}\left[\ln \left(T_{-c, c}^{\theta}+\frac{1}{2 \lambda}\right)\right] .
\end{aligned}
$$


Thus,

$$
2 \lambda \mathbf{E}\left[T_{c}^{(\lambda)}\right]-\ln (2 \lambda) \underset{\lambda \rightarrow \infty}{\longrightarrow} \mathbf{E}\left[\ln T_{-c, c}^{\theta}\right],
$$

which is precisely (67). Moreover, by the integral representation (49) for $\mathbf{E}\left[\ln T_{-c, c}^{\theta}\right]$, we deduce (68).

Case 2: $\lambda$ small. We shall now study the case $\lambda \rightarrow 0$. We have

$$
T_{c}^{(\lambda)}=\frac{1}{2 \lambda} \ln \left(1+2 \lambda T_{-c, c}^{\theta}\right) .
$$

For $c<\pi / 8$, from (3), (at least) the first two positive moments of $T_{-c, c}^{\theta}$ are finite: $\mathbf{E}\left[\left(T_{-c, c}^{\theta}\right)^{p}\right]<\infty(p=1,2)$. We make the elementary computation

$$
\begin{aligned}
\frac{1}{\lambda}\left(\frac{\ln (1+2 \lambda x)}{2 \lambda}-x\right) & =\frac{1}{\lambda}\left(\frac{1}{2 \lambda} \int_{1}^{1+2 \lambda x} \frac{d y}{y}-x\right) \\
& \stackrel{y=1+a}{=} \frac{1}{2 \lambda^{2}} \int_{0}^{2 \lambda x}\left(\frac{1}{1+a}-1\right) d a \stackrel{a=2 \lambda b}{=}-2 \int_{0}^{x} \frac{b d b}{1+2 \lambda b} \stackrel{\lambda \rightarrow 0}{\longrightarrow}-x^{2} .
\end{aligned}
$$

Consequently, by replacing $x=T_{-c, c}^{\theta}$, we have

$$
\frac{1}{\lambda}\left(\mathbf{E}\left[T_{c}^{(\lambda)}\right]-\mathbf{E}\left[T_{-c, c}^{\theta}\right]\right)=\mathbf{E}\left[-2 \int_{0}^{T_{-c, c}^{\theta}} \frac{b d b}{1+2 \lambda b}\right]
$$

We may now use the dominated convergence theorem [7], since the $(d b)$ integral is majorized by $\left(T_{-c, c}^{\theta}\right)^{2}$, which is integrable. Thus

$$
\frac{1}{\lambda}\left(\mathbf{E}\left[T_{c}^{(\lambda)}\right]-\mathbf{E}\left[T_{-c, c}^{\theta}\right]\right) \underset{\lambda \rightarrow 0}{\longrightarrow}-\mathbf{E}\left[\left(T_{-c, c}^{\theta}\right)^{2}\right] .
$$

Following the proof of Proposition 2.7 and using Bougerol's identity and the scaling property we obtain

$$
\mathbf{E}\left[\left(\operatorname{sh} B_{t}\right)^{2}\right]=\mathbf{E}\left[\delta_{A_{t}}^{2}\right]=\mathbf{E}\left[A_{t}\left(\delta_{1}\right)^{2}\right]=\mathbf{E}\left[A_{t}\right] \mathbf{E}\left[\left(\delta_{1}\right)^{2}\right]=\mathbf{E}\left[A_{t}\right] .
$$

Thus, for $t=T_{-c, c}^{\gamma}$, we have

$$
\mathbf{E}\left[A_{T_{-c, c}^{\gamma}}\right]=\mathbf{E}\left[\left(\operatorname{sh} B_{T_{-c, c}^{\gamma}}\right)^{2}\right] .
$$

Similarly,

$$
\mathbf{E}\left[\left(\operatorname{sh} B_{t}\right)^{4}\right]=\mathbf{E}\left[\left(\delta_{A_{t}}\right)^{4}\right]=\mathbf{E}\left[\left(A_{t}\right)^{2}\left(\delta_{1}\right)^{4}\right]=\mathbf{E}\left[\left(A_{t}\right)^{2}\right] \mathbf{E}\left[\left(\delta_{1}\right)^{4}\right]=3 \mathbf{E}\left[\left(A_{t}\right)^{2}\right] .
$$

Thus, for $t=T_{-c, c}^{\gamma}$, we have

$$
\mathbf{E}\left[\left(A_{T_{-c, c}^{\gamma}}\right)^{2}\right]=\frac{1}{3} \mathbf{E}\left[\left(\operatorname{sh} B_{T_{-c, c}^{\gamma}}\right)^{4}\right] .
$$


So, because $A_{T_{-c, c}^{\gamma}}=T_{-c, c}^{\theta}$, we deduce (69). In order to prove (70), it suffices to remark that

$$
\mathbf{E}\left[T_{c}^{(0)}\right]=\mathbf{E}\left[T_{-c, c}^{\theta}\right]=\mathbf{E}\left[A_{T_{-c, c}^{\gamma}}\right]=\mathbf{E}\left[\left(\operatorname{sh} B_{T_{-c, c}^{\gamma}}\right)^{2}\right] .
$$

On the one hand, by using the density of $B_{T_{-c, c}^{\gamma}}$ :

$$
\begin{aligned}
\mathbf{E}\left[\left(\operatorname{sh} B_{T_{-c, c}^{\gamma}}\right)^{4}\right] & =\int_{-\infty}^{\infty} \frac{d y}{2 c} \frac{1}{\operatorname{ch}(\pi y /(2 c))}(\operatorname{sh} y)^{4} \\
& =\int_{0}^{\infty} \frac{d y}{c} \frac{1}{\operatorname{ch}(\pi y /(2 c))}(\operatorname{sh} y)^{4} \\
& \stackrel{z=y / c}{=} \int_{0}^{\infty} d z \frac{1}{\operatorname{ch}(\pi z / 2)}(\operatorname{sh}(c z))^{4}
\end{aligned}
$$

which is finite if and only if $c<\pi / 8$. In order to prove this, it suffices to use the standard expressions: $\operatorname{sh} x=\left(e^{x}-e^{-x}\right) / 2$ and $\operatorname{ch} x=\left(e^{x}+e^{-x}\right) / 2$. On the other hand (note that $T \equiv T_{-c, c}^{\gamma}$ ), we remark that $-B_{T} \stackrel{\text { law }}{=} B_{T}$ and [31, Exercise 3.10], $\mathbf{E}\left[e^{k B_{T}}\right]=\mathbf{E}\left[e^{k^{2} T / 2}\right]=1 / \cos (k c)$, for $0 \leqslant k<\pi(2 c)^{-1}$, thus

$$
\begin{aligned}
\mathbf{E}\left[\left(\operatorname{sh} B_{T}\right)^{4}\right] & =\frac{1}{2^{4}} \mathbf{E}\left[\left(e^{B_{T}}-e^{-B_{T}}\right)^{4}\right] \\
& =\frac{1}{2^{4}} \mathbf{E}\left[e^{4 B_{T}}-4 e^{3 B_{T}-B_{T}}+6 e^{2 B_{T}-2 B_{T}}-4 e^{B_{T}-3 B_{T}}+e^{-4 B_{T}}\right] \\
& =\frac{1}{2^{4}}\left(2 \mathbf{E}\left[e^{4 B_{T}}\right]-8 E\left[e^{2 B_{T}}\right]+6\right) \\
& =\frac{1}{2^{3}}\left(\frac{1}{\cos (4 c)}-4 \frac{1}{\cos (2 c)}+3\right),
\end{aligned}
$$

which is precisely (72) and this is finite if and only if $c<\pi / 8$. Moreover, asymptotically for $c \rightarrow 0$, by using the scaling property, we have

$$
\begin{aligned}
\mathbf{E}\left[\left(\operatorname{sh} B_{T_{-c, c}^{\gamma}}\right)^{4}\right] & =\mathbf{E}\left[\left(\operatorname{sh}\left(c B_{T_{-1,1}^{\gamma}}\right)\right)^{4}\right] \underset{c \rightarrow 0}{\simeq} c^{4} \mathbf{E}\left[\left(B_{T_{-1,1}^{\gamma}}\right)^{4}\right] \\
& =c^{4} \cdot 3 \mathbf{E}\left[\left(T_{-1,1}^{\gamma}\right)^{2}\right]=c^{4} \cdot 3 \frac{5}{3}=5 c^{4},
\end{aligned}
$$

since $\mathbf{E}\left[\left(T_{-1,1}^{\gamma}\right)^{2}\right]=5 / 3$ (see [30]; by using the notation of this paper, Table 3 : $\mathbf{E}\left[X_{t}^{2}\right]=t(2+3 t) / 3$ for $X_{t}=C_{1}$ and $\left.t=1\right)$. This asymptotics can also be obtained from (72) by developing $\cos (4 c)$ and $\cos (2 c)$ into series up to the second-order term and keeping the terms of the order $c^{4}$.

$\mathrm{R} \mathrm{e} \mathrm{m}$ a $\mathrm{r} \mathrm{k}$. If we slightly modify the above study for the OrnsteinUhlenbeck process by inserting a diffusion coefficient $D$ :

$$
Z_{t}=z_{0}+\sqrt{2 D} \widetilde{Z}_{t}-\lambda \int_{0}^{t} Z_{s} d s
$$


we obtain

$$
Z_{t}=e^{-\lambda t}\left(z_{0}+\sqrt{2 D} \int_{0}^{t} e^{\lambda s} d \widetilde{Z_{s}}\right)=e^{-\lambda t} \mathbf{B}_{\alpha_{t}}
$$

where in the second equation we used the Dambis-Dubins-Schwarz theorem with

$$
\alpha_{t}=2 D \int_{0}^{t} e^{2 \lambda s} d s=D \frac{e^{2 \lambda t}-1}{\lambda} \Rightarrow \alpha_{t}^{-1}=\frac{1}{2 \lambda} \ln \left(1+\frac{\lambda}{D} t\right) .
$$

Thus,

$$
2 \lambda \mathbf{E}\left[T_{c}^{(\lambda)}\right]-\ln \frac{\lambda}{D} \underset{\lambda \rightarrow \infty}{\longrightarrow} \mathbf{E}\left[\ln T_{-c, c}^{\theta}\right]
$$

because

$$
\begin{aligned}
\mathbf{E}\left[T_{c}^{(\lambda)}\right] & =\frac{1}{2 \lambda} \mathbf{E}\left[\ln \left(1+\frac{\lambda}{D} T_{-c, c}^{\theta}\right)\right]=\frac{1}{2 \lambda} \mathbf{E}\left[\ln \left(\frac{\lambda}{D}\left(T_{-c, c}^{\theta}+\frac{D}{\lambda}\right)\right)\right] \\
& =\frac{\ln (\lambda / D)}{2 \lambda}+\frac{1}{2 \lambda} \mathbf{E}\left[\ln \left(T_{-c, c}^{\theta}+\frac{D}{\lambda}\right)\right] .
\end{aligned}
$$

Moreover,

$$
\begin{aligned}
\mathbf{E}\left[\ln T_{-c, c}^{\theta}\right] & =2 \ln z_{0}+\mathbf{E}\left[\ln T_{-c, c}^{\theta^{(1)}}\right] \\
& =2 \ln z_{0}+\int_{0}^{\infty} \frac{d z}{\operatorname{ch}(\pi z / 2)} \ln \operatorname{sh}(c z)+\ln 2+c_{\mathbf{E}}
\end{aligned}
$$

where $T_{-c, c}^{\theta^{(1)}}$ denotes the first hitting time of the symmetric conic boundary of angle $c$ for a Brownian motion $Z$ starting from 1 .

For $\lambda$ small, we replace $2 T_{-c, c}^{\theta}$ by $\left(z_{0}^{2} D\right) T_{-c, c}^{\theta}$ in the proof of Proposition 3.3 ( $\lambda$ small case) and we have

$$
T_{c}^{(\lambda)}=\frac{1}{2 \lambda} \ln \left(1+\lambda \frac{z_{0}^{2}}{D} T_{-c, c}^{\theta}\right) .
$$

By repeating the previous calculation, we make the elementary computation:

$$
\frac{1}{\lambda}\left(\frac{\ln \left(1+\left(z_{0}^{2} / D\right) x\right)}{2 \lambda}-\frac{z_{0}^{2}}{D} x\right)=-\frac{1}{2} \int_{0}^{x} \frac{\left(z_{0}^{2} / D\right)^{2} b d b}{1+\lambda z_{0}^{2} / D b} \underset{\lambda \rightarrow 0}{\longrightarrow}-\left(\frac{z_{0}^{2}}{2 D}\right)^{2} x^{2} .
$$

We replace $x=T_{-c, c}^{\theta}$, and by the dominated convergence theorem [7], for $c<\pi / 8$, we obtain

$$
\begin{aligned}
\frac{1}{\lambda}\left(\mathbf{E}\left[T_{c}^{(\lambda)}\right]-\frac{z_{0}^{2}}{2 D} \mathbf{E}\left[\left(\operatorname{sh~} B_{T_{-c, c}^{\gamma}}\right)^{2}\right]\right) & \underset{\lambda \rightarrow 0}{\longrightarrow}-\frac{1}{3}\left(\frac{z_{0}^{2}}{2 D}\right)^{2} \mathbf{E}\left[\left(T_{-c, c}^{\theta}\right)^{2}\right] \\
& =-\frac{1}{3}\left(\frac{z_{0}^{2}}{2 D}\right)^{2} \mathbf{E}\left[\left(\operatorname{sh~} B_{T_{-c, c}^{\gamma}}\right)^{4}\right],
\end{aligned}
$$

where $\mathbf{E}\left[\left(\operatorname{sh} B_{T_{-c, c}^{\gamma}}\right)^{4}\right]$ is given by (71), (72) and asymptotically, as $c \rightarrow 0$ by (73). 
Acknowledgments. I would like to thank Alain Comtet for his contribution in the proof of Proposition 2.6 and the Human Frontier Science Program for its support. This article represents the first part of my $\mathrm{PhD}$ thesis, under the supervision of Professors D. Holcman and M. Yor.

\section{REFERENCES}

1. Абрамовии М., Стиган И. Справочник по специальным функциям с формулами, графиками и математическими таблицами. М.: Наука, 1979, 830 с.

2. Alili L., Dufresne D., Yor M. Sur l'identité de Bougerol pour les fonctionnelles exponentielles du mouvement brownien avec drift. - Exponential Functionals and Principal Values Related to Brownian Motion. Madrid: Univ. Autónoma de Madrid, 1997, p. 3-14.

3. André D. Solution directe du problème résolu par M. Bertrand. - C.R. Acad. Sci. Paris, 1887, v. 105, p. 436-437.

4. Bentkus V., Pap G., Yor M. Optimal bounds for Cauchy approximations for the winding distribution of planar Brownian motion. - J. Theoret. Probab., 2003, v. 16, № 2 , p. 345-361.

5. Bertoin J., Werner $W$. Asymptotic windings of planar Brownian motion revisited via the Ornstein-Uhlenbeck process. - Lecture Notes in Math., 1994, v. 1583, p. 138152.

6. Biane P., Yor M. Valeurs principales associées aux temps locaux browniens. - Bull. Sci. Math., 1987, v. 111, № 1, p. 23-101.

7. Billingsley P. Probability and Measure. 3rd ed. New York: Wiley, 1995, 593 p.

8. Bougerol Ph. Exemples de théorèmes locaux sur les groupes résolubles. - Ann. Inst. H. Poincaré, 1983, v. 19, № 4, p. 369-391.

9. Burkholder D. Exit times of Brownian motion, harmonic majorization, and Hardy spaces. - Adv. in Math., 1977, v. 26, № 2, p. 182-205.

10. Chaumont L., Yor M. Exercises in Probability: A Guided Tour from Measure Theory to Random Processes, via Conditioning. Cambridge: Cambridge Univ. Press, 2003, $236 \mathrm{p}$.

11. Comtet A., Monthus C., Yor M. Exponential functionals of Brownian motion and disordered systems. - J. Appl. Probab., 1998, v. 35, № 2, p. 255-271.

12. Donati-Martin C., Matsumoto H., Yor M. On positive and negative moments of the integral of geometric Brownian motions. - Statist. Probab. Lett., 2000, v. 49, p. 4552 .

13. Dufresne D. Laguerre series for Asian and other options. - Math. Finance, 2000, v. 10, № 4, p. 407-428.

14. Dufresne D., Yor M. A two-dimensional extension of Bougerol's identity in law for the exponential functional of Brownian motion: the story so far (в печати).

15. Durrett $R$. A new proof of Spitzer's result on the winding of 2-dimensional Brownian motion. - Ann. Probab., 1982, v. 10, № 1, p. 244-246.

16. Gallardo L. Mouvement brownien et calcul d'Itô. Paris: Hermann, 2008, 240 p.

17. Градштейн И. С., Рыжик И. М. Таблицы интегралов, рядов и произведений. М.: Наука, 1971, 1108 с.

18. Ishiyama $K$. Methods for evaluating density functions of exponential functionals represented as integrals of geometric Brownian motion. - Methodol. Comput. Appl. Probab., 2005, v. 7, № 3, p. 271-283.

19. Ито К., Маккин Г. Диффузионные процессы и их траектории. М.: Мир, 1968, $394 \mathrm{c}$.

20. Le Gall J. F. Some properties of planar Brownian motion. - Lecture Notes in Math., 1992, v. 1527 , p. 111-235. 
21. Le Gall J.F., Yor M. Etude asymptotique des enlacements du mouvement brownien autour des droites de l'espace. - Probab. Theory Related Fields, 1987, v. 74, p. 617635.

22. Лебедев Н. Н. Специальные функции и их приложения. М.-Л.: Физматгиз, 1963, $358 \mathrm{c.}$

23. Lévy P. Random functions: general theory with special reference to Laplacian random functions. - Euvres de Paul Lévy, v. IV. Ed. by D. Dugué et al. Paris: GauthierVillars, 1980, paper № 158.

24. Mansuy R., Yor M. Aspects of Brownian Motion. Berlin: Springer-Verlag, 2008, 195 p.

25. Matsumoto H., Yor M. On Bougerol and Dufresne's identities for exponential Brownian functionals. - Proc. Japan Acad. Ser. A Math. Sci., 1998, v. 74, № 10, p. 152-155.

26. Matsumoto H., Yor M. Exponential functionals of Brownian motion. I: Probability laws at fixed time. - Probab. Surveys, 2005, v. 2, p. 312-347.

27. Messulam P., Yor M. On D. Williams' «pinching method» and some applications. J. London Math. Soc., 1982, v. 26, p. 348-364.

28. Pap G., Yor M. The accuracy of Cauchy approximation for the windings of planar Brownian motion. - Period. Math. Hungar., 2000, v. 41, № 1-2, p. 213-226.

29. Pitman J. W., Yor M. Asymptotic laws of planar Brownian motion. - Ann. Probab., 1996, v. 14, p. 733-779.

30. Pitman J. W., Yor $M$. Infinitely divisible laws associated with hyperbolic functions. Canad. J. Math., 2003, v. 55, № 2, p. 292-330.

31. Revuz D., Yor M. Continuous Martingales and Brownian Motion. Berlin: Springer, 1999, $602 \mathrm{p}$.

32. Spitzer F. Some theorems concerning two-dimensional Brownian motion. - Trans. Amer. Math. Soc., 1958, v. 87, p. 187-197.

33. Vallois P. Amplitude du mouvement Brownien et juxtaposition des excursions positives et negatives. - Lecture Notes in Math., 1992, v. 1526, p. 361-373.

34. Williams D. A simple geometric proof of Spitzer's winding number formula for 2dimensional Brownian motion. Swansea: University College, 1974 (preprint).

35. Yor M. Loi de l'indice du lacet Brownien et distribution de Hartman-Watson. - Z Wahrscheinlichkeitstheor. verw. Geb., 1980, v. 53, № 1, p. 71-95.

36. Yor $M$. Une décomposition asymptotique du nombre de tours du mouvement brownien complexe. - Astérisque, 1985, № 132, p. 103-126.

37. Yor M. Generalized meanders as limits of weighted Bessel processes, and an elementary proof of Spitzer's asymptotic result on Brownian windings. - Studia Sci. Math. Hungar., 1997, v. 33, № 1-3, p. 339-343.

38. Yor M. Exponential Functionals of Brownian Motion and Related Processes. Berlin: Springer-Verlag, 2001, 205 p. 